\title{
IFSS, TG, FT-IR spectra of impregnated sugar palm (Arenga pinnata) fibres and mechanical properties of their composites.
}

\begin{abstract}
This study aimed to investigate the effect of resin impregnation on the interfacial shear strength (IFSS), thermogravimetric (TG) and fourier transform infrared (FT-IR) of sugar palm (Arenga pinnata) fibres. In addition, the effect of resin impregnation on the mechanical properties of sugar palm fibre reinforced unsaturated polyester (UP) composites was also studied. The fibres were impregnated with UP via vacuum resin impregnation process at a pressure of $600 \mathrm{mmHg}$ for $5 \mathrm{~min}$. Composites of 10, 20, 30, 40 and $50 \%$ fibre loadings were fabricated and tested for tensile and flexural properties. It was observed that the impregnation process caused the fibres to be enclosed by UP resin and this gave a strong influence to the increase of its interfacial bonding by the increase of its IFSS from single fibre pull-out test. It was also observed with TG and FT-IR spectra that the impregnated fibre had lower moisture uptake than the control and there was no significant increase in thermal stability of the impregnated fibre. The sequence of fibre decomposition started from the evaporation of moisture, hemicelluloses, cellulose, lignin and finally ash content and the presence of these components were proven by FT-IR spectra. For the composite specimens, due to the high interfacial bonding of the impregnated fibre and the matrix, the impregnated composites showed consistently higher tensile strength, tensile modulus, elongation at break, flexural strength, flexural modulus and toughness than the control samples. It was also observed that $30 \%$ fibre loading gave optimum properties.
\end{abstract}

Keyword: Impregnation modification; Sugar palm fibre; Vacuum resin impregnation. 